\title{
A Fractional Trust Region Method for Linear Equality Constrained Optimization
}

\author{
Honglan Zhu, ${ }^{1,2}$ Qin Ni, ${ }^{1}$ Liwei Zhang, ${ }^{3}$ and Weiwei Yang ${ }^{1}$ \\ ${ }^{1}$ College of Science, Nanjing University of Aeronautics and Astronautics, Nanjing 210016, China \\ ${ }^{2}$ Business School, Huaiyin Institute of Technology, Huaian 223003, China \\ ${ }^{3}$ Jiangsu Cable, Nanjing 210000, China
}

Correspondence should be addressed to Honglan Zhu; zhuhonglan1981@163.com

Received 21 April 2016; Accepted 29 May 2016

Academic Editor: Josef Diblík

Copyright (C) 2016 Honglan Zhu et al. This is an open access article distributed under the Creative Commons Attribution License, which permits unrestricted use, distribution, and reproduction in any medium, provided the original work is properly cited.

\begin{abstract}
A quasi-Newton trust region method with a new fractional model for linearly constrained optimization problems is proposed. We delete linear equality constraints by using null space technique. The fractional trust region subproblem is solved by a simple dogleg method. The global convergence of the proposed algorithm is established and proved. Numerical results for test problems show the efficiency of the trust region method with new fractional model. These results give the base of further research on nonlinear optimization.
\end{abstract}

\section{Introduction}

In this paper, we consider the linear equality constrained optimization problem:

$$
\begin{array}{ll}
\min _{x \in R^{n}} & f(x), \\
\text { s.t. } & A^{T} x=\mathfrak{b},
\end{array}
$$

where $f(x)$ are continuously differentiable, $A \in R^{n \times m}, \mathfrak{b} \in$ $R^{m}$, and $\operatorname{rank}(A)=m$.

Trust region methods have the advantage in the theoretical analysis of convergence properties and the practice. Besides, Davidon proposed a conic model which makes use of more incorporate information at each iteration (see [1]). It is believed that combing trust region techniques with a conic model would be appealing. And it has attracted more and more attention of the researchers (see [2-8]).

For unconstrained optimization problems, we proposed a new fractional model (see [9]):

$$
\begin{aligned}
\psi_{k}(s)= & \frac{\left(1+c_{k}^{T} s\right)}{\left(1-a_{k}^{T} s\right)\left(1-b_{k}^{T} s\right)} g_{k}^{T} s \\
& +\frac{1}{2} \frac{\left(1+c_{k}^{T} s\right)^{2}}{\left(1-a_{k}^{T} s\right)^{2}\left(1-b_{k}^{T} s\right)^{2}} s^{T} B_{k} s,
\end{aligned}
$$

where $a_{k}, b_{k}, c_{k} \in R^{n}$ are horizontal vectors, $g_{k}=\nabla f\left(x_{k}\right), s \in$ $R^{n}, B_{k} \in R^{n \times n}$ is symmetric and an approximate Hessian of $f(x)$ at $x_{k}$. Then, the trust region subproblem of the unconstrained optimization problems is

$$
\begin{array}{ll}
\min & \psi_{k}(s), \\
\text { s.t. } & \|s\| \leqslant \Delta_{k}, \\
& \left|\left(1-a_{k}^{T} s\right)\left(1-b_{k}^{T} s\right)\right| \geqslant \epsilon_{0},
\end{array}
$$

where $\epsilon_{0}\left(0<\epsilon_{0}<1\right)$ is a sufficiently small positive number, $\|\cdot\|$ refers to the Euclidean norm, and $\Delta_{k}>0$ is a trust region radius. If $b_{k}=c_{k}=0$, then $\psi_{k}(s)$ is reduced to the conic model. If $a_{k}=b_{k}=c_{k}=0$, then $\psi_{k}(s)$ is the quadratic model. In order to ensure that the fractional model function $\psi_{k}(s)$ is bounded over the trust region $\left\{s \mid\|s\| \leqslant \Delta_{k}\right\}$, we assume $\exists \epsilon_{1} \in(0,1 / 3)$ :

$$
\begin{gathered}
\left\|a_{k}\right\| \Delta_{k} \leqslant \epsilon_{1}, \\
\left\|b_{k}\right\| \Delta_{k} \leqslant \epsilon_{1}, \\
\left\|c_{k}\right\| \Delta_{k} \leqslant \epsilon_{1} .
\end{gathered}
$$

We denote

$$
\widetilde{\Delta}_{k}=\min \left\{\Delta_{k}, \frac{\epsilon_{1}}{\left\|a_{k}\right\|}, \frac{\epsilon_{1}}{\left\|b_{k}\right\|}, \frac{\epsilon_{1}}{\left\|c_{k}\right\|}\right\}
$$


then, (4) reduces to a simplified fractional trust region subproblem:

$$
\begin{array}{ll}
\min & \psi(s), \\
\text { s.t. } & \|s\| \leqslant \widetilde{\Delta}_{k} .
\end{array}
$$

However, the subproblem (7) is solved only in quasi-Newton direction in [9]. In this paper, we made a further research, where the subproblem (7) is solved by a generalized dogleg algorithm.

For linear equality constraints problem, we focus on the problems which are solved by a trust region method with new fractional model. If the constraints are linear inequality constraints or some constraint functions are nonlinear, then a few difficulties may arise. However, for these cases, the linear equality constraints problem may be as the subproblems of them. For example, the inequality constraints can be removed by an active set technique or a barrier transformation, and then the nonlinear constraints are linearized.

In [10], Sun et al. established the algorithm for the problem (1)-(2) and prove the convergence. However, they do not consider the model computation. In [11], Lu and $\mathrm{Ni}$ proposed a trust region method with new conic model for solving (1)-(2) and carried out the numerical experiments.

In this paper, we use a simple dogleg method to solve fractional model subproblems and present a quasi-Newton trust region algorithm for solving linear equality constrained optimization problems. This is a continuing work of the fractional model (see [9]), where the linear equality constraints (2) are deleted by using null space techniques.

This paper is organized as follows. In Section 2, we give a description of the fractional trust region subproblem. In Section 3, we give a generalized dogleg algorithm for solving the fractional subproblem. In Section 4, we propose a new quasi-Newton method based on the fractional model for solving linearly constrained optimization problems and prove the global convergence of the proposed method under the reasonable assumptions. The numerical results are presented in Section 5.

\section{The Fractional Trust Region Subproblem}

In order to solve the problem (1)-(2), we assume that $A$ is column full rank and constraints are consistent. That is, the current point $x_{k}$ always satisfies $A^{T} x_{k}=\mathfrak{b}$. Obviously, the constrained condition is equivalent to $A^{T} s=0$ if $x=x_{k}+s$. Therefore, combing with (1)-(4), we can obtain that the trial step $s_{k}$ is computed by the following subproblem:

$$
\begin{array}{ll}
\min & \psi_{k}(s), \\
\text { s.t. } & A^{T} s=0, \\
& \left|\left(1-a_{k}^{T} s\right)\left(1-b_{k}^{T} s\right)\right| \geqslant \epsilon_{0}, \\
& \|s\| \leqslant \Delta_{k} .
\end{array}
$$

It can be found that our trust region subproblem (8)-(11) is the minimization of a fractional function subject to the trust region constraint and the linear constraints.
In order to solve (8)-(11), firstly we consider removing the constraint (10) by the same assumption as in [9]. That is, we assume the parameters $a_{k}, b_{k}$, and $c_{k}$ satisfied (5); then, subproblem (8)-(11) can be rewritten as the following reduced subproblem:

$$
\begin{array}{ll}
\min & \psi_{k}(s) \\
\text { s.t. } & A^{T} s=0, \\
& \|s\| \leqslant \widetilde{\Delta}_{k},
\end{array}
$$

where $\widetilde{\Delta}_{k}$ is defined as (6).

The null space technology (see $[4,12,13])$ is an important technique for solving equality constraints programming problems. In the following, we use this technology to eliminate constraint (13). Since $A$ has full column rank, then there exist an orthogonal matrix $Q \in R^{n \times n}$ and a nonsingular upper triangular matrix $R \in R^{m \times m}$ such that

$$
A=Q R=\left[\begin{array}{ll}
Q_{1} & Q_{2}
\end{array}\right]\left[\begin{array}{c}
R_{1} \\
0
\end{array}\right]=Q_{1} R_{1}
$$

where $Q_{1} \in R^{n \times m}, Q_{2} \in R^{n \times(n-m)}$, and $R_{1} \in R^{m \times m}$. Then, (13) can be rewritten as

$$
R_{1}^{T} Q_{1}^{T} s=0
$$

Therefore, the feasible point for (13) can be presented by

$$
s=Q_{2} u
$$

for any $u \in R^{n-m}$, where $Q_{2} u$ lies in the null space of $A$. Then, the subproblem (12)-(14) becomes

$$
\begin{aligned}
\min \tilde{\psi}_{k}(u) & \\
= & \frac{\left(1+\widetilde{c}_{k}^{T} u\right) \tilde{g}_{k}^{T} u}{\left(1-\tilde{a}_{k}^{T} u\right)\left(1-\widetilde{b}_{k}^{T} u\right)} \\
& +\frac{\left(1+\widetilde{c}_{k}^{T} u\right)^{2} u^{T} \widetilde{B}_{k} u}{2\left(1-\widetilde{a}_{k}^{T} u\right)^{2}\left(1-\widetilde{b}_{k}^{T} u\right)^{2}},
\end{aligned}
$$$$
\text { s.t. }\|u\| \leqslant \widetilde{\Delta}_{k} \text {, }
$$

where $\tilde{a}_{k}, \widetilde{b}_{k}$, and $\widetilde{c}_{k}$ are reduced horizontal vectors, $\widetilde{g}_{k}$ is the reduced gradient, $\widetilde{B}_{k}$ is Hessian approximation, and

$$
\begin{aligned}
& \tilde{g}_{k}=Q_{2}^{T} g_{k}, \\
& \widetilde{B}_{k}=Q_{2}^{T} B_{k} Q_{2}, \\
& \tilde{a}_{k}=Q_{2}^{T} a_{k}, \\
& \tilde{b}_{k}=Q_{2}^{T} b_{k}, \\
& \widetilde{c}_{k}=Q_{2}^{T} c_{k} .
\end{aligned}
$$


It can be seen that this subproblem has the same form as the subproblem (7) of the unconstrained optimization problems and it can be considered as the subproblem of the unconstrained minimization over $u$. Therefore, we can find a solution of (18)-(19) by the dogleg method. Besides, it is easy to find that $\|s\|=\left\|Q_{2} u\right\| \leqslant \widetilde{\Delta}_{k}$ is equivalent to $\|u\| \leqslant \widetilde{\Delta}_{k}$ due to $Q_{2}^{T} Q_{2}=I$.

\section{The Dogleg Method of Fractional Trust Region Subproblem}

Now we consider calculating the trial step $u_{k}$ of the new subproblem (18)-(19) by a simple dogleg method. Firstly, we need to recall the choice of the Newton point $u_{N}$ as the following subalgorithm (see [9]).

Subalgorithm 3.1. Given $B_{k}>0$,

$$
\epsilon_{1} \in\left(0, \frac{1}{3}\right)
$$

Step 1. Calculate $\widetilde{a}_{k}, \widetilde{b}_{k}, \widetilde{c}_{k}, \widetilde{g}_{k}$, and $\widetilde{B}_{k}$ as defined in (20) and

$$
\begin{aligned}
\xi_{a} & =\widetilde{c}_{k}^{T} \widetilde{B}_{k}^{-1} \widetilde{g}_{k}+\widetilde{a}_{k}^{T} \widetilde{B}^{-1} \widetilde{g}_{k} \widetilde{b}_{k}^{T} \widetilde{B}_{k}^{-1} \widetilde{g}_{k}, \\
\xi_{b} & =\widetilde{a}_{k}^{T} \widetilde{B}_{k}^{-1} \widetilde{g}_{k}+\widetilde{b}_{k}^{T} \widetilde{B}_{k}^{-1} \widetilde{g}_{k}-1 .
\end{aligned}
$$

Step 2. If $\xi_{a}<0$, then $u_{N}=-\tau_{N} \widetilde{B}_{k}^{-1} \widetilde{g}_{k}$, where

$$
\tau_{N}=\frac{\xi_{b}+\sqrt{\xi_{b}^{2}+\left(-4 \xi_{a}\right)}}{-2 \xi_{a}} .
$$

Step 3. If $\xi_{a} \geqslant 0$, then set $\widetilde{b}_{k}=\widetilde{c}_{k}=0$. If $\left|\widetilde{a}_{k}^{T} \widetilde{B}_{k}^{-1} \widetilde{g}_{k}\right| \leqslant \epsilon_{1}$, then $u_{N}=-\widetilde{B}_{k}^{-1} \widetilde{g}_{k} /\left(1-\widetilde{a}_{k}^{T} \widetilde{B}_{k}^{-1} \widetilde{g}_{k}\right)$. If $\left|\widetilde{a}_{k}^{T} \widetilde{B}_{k}^{-1} \widetilde{g}_{k}\right|>\epsilon_{1}$, then set $\widetilde{a}_{k}=0$ and $u_{N}=-\widetilde{B}_{k}^{-1} \widetilde{g}_{k}$.

In the following, we consider determining the steepest descent point of (18)-(19), where the steepest descent point is defined by Definition 1 . Let $u=-\tau \widetilde{g}_{k}\left(\widetilde{g}_{k} \neq 0\right)$ and from (18) we have

$$
\widehat{\psi}(\tau)=\widetilde{\psi}_{k}\left(-\tau \widetilde{g}_{k}\right)
$$

then, (18)-(19) becomes

$$
\begin{array}{ll}
\min & \widehat{\psi}(\tau), \\
\text { s.t. } & 0 \leqslant \tau \leqslant \tau_{\Delta},
\end{array}
$$

where

$$
\begin{aligned}
& \widehat{\psi}(\tau) \\
& =\frac{\tau\left(1-\tau \widetilde{c}_{k}^{T} \widetilde{g}_{k}\right)\left(-2 v_{\tau} \widetilde{g}_{k}^{T} \widetilde{g}_{k}+\tau\left(1-\tau \widetilde{c}_{k}^{T} \widetilde{g}_{k}\right) \widetilde{g}_{k}^{T} \widetilde{B}_{k} \widetilde{g}_{k}\right)}{2 v_{\tau}^{2}}, \\
& v_{\tau}=\left(1+\tau \widetilde{a}_{k}^{T} \widetilde{g}_{k}\right)\left(1+\tau \widetilde{b}_{k}^{T} \widetilde{g}_{k}\right), \\
& \tau_{\Delta}=\frac{\widetilde{\Delta}_{k}}{\left\|\widetilde{g}_{k}\right\|} .
\end{aligned}
$$

Definition 1. Let $\tau_{\mathrm{cp}}$ be the solution of (25). Then, $u_{\mathrm{cp}}=-\tau_{\mathrm{cp}} g$ is called a steepest descent point of (18)-(19).

In order to discuss the stationary points of $\widehat{\psi}(\tau)$, by computation we have that the derivative of $\widehat{\psi}(\tau)$ is

$$
\widehat{\psi}^{\prime}(\tau)=\frac{\varphi_{\tau} \widetilde{\varphi}_{\tau}}{v_{\tau}^{3}},
$$

where

$$
\begin{aligned}
& \varphi_{\tau}=a_{\tau} \tau^{2}+b_{\tau} \tau+\tilde{g}_{k}^{T} \widetilde{g}_{k}, \\
& \widetilde{\varphi}_{\tau}=\tilde{a}_{\tau} \tau^{2}+2 \widetilde{c}_{k}^{T} \widetilde{g}_{k} \tau-1, \\
& a_{\tau}=\widetilde{c}_{k}^{T} \widetilde{g}_{k} \widetilde{g}_{k}^{T} \widetilde{B}_{k} \widetilde{g}_{k}+\widetilde{a}_{k}^{T} \widetilde{g}_{k} \widetilde{b}_{k}^{T} \widetilde{g}_{k} \widetilde{g}_{k}^{T} \widetilde{g}_{k}, \\
& b_{\tau}=\tilde{a}_{k}^{T} \widetilde{g}_{k} \widetilde{g}_{k}^{T} \widetilde{g}_{k}+\widetilde{b}_{k}^{T} \widetilde{g}_{k} \tilde{g}_{k}^{T} \widetilde{g}_{k}-\widetilde{g}_{k}^{T} \widetilde{B}_{k} \widetilde{g}_{k}, \\
& \widetilde{a}_{\tau}=\widetilde{c}_{k}^{T} \widetilde{g}_{k} \widetilde{a}_{k}^{T} \widetilde{g}_{k}+\widetilde{c}_{k}^{T} \widetilde{g}_{k} \widetilde{b}_{k}^{T} \widetilde{g}_{k}+\widetilde{a}_{k}^{T} \widetilde{g}_{k} \widetilde{b}_{k}^{T} \widetilde{g}_{k} .
\end{aligned}
$$

By direct calculating, we have

$$
a_{\tau}=\widetilde{a}_{\tau} \widetilde{g}_{k}^{T} \widetilde{g}_{k}-b_{\tau} \widetilde{c}_{k}^{T} \widetilde{g}_{k}
$$

During the analysis, we find that if $b_{\tau} \geqslant 0$ and $\widetilde{c}_{k}^{T} \widetilde{g}_{k} \leqslant 0$, then (29) and (30) may have no positive real zero points in most of the cases, respectively. Thus, in order to simplify the discussion, we assume

$$
\begin{aligned}
b_{\tau} & <0, \\
\widetilde{c}_{k}^{T} \widetilde{g}_{k} & >0 .
\end{aligned}
$$

In order to discuss the feasible stationary points of $\varphi_{\tau}$ and $\widetilde{\varphi}_{\tau}$, we first define the sets $T_{\varphi}$ and $T_{\widetilde{\varphi}}$ which contain all the positive extremum points of $\varphi_{\tau}$ and $\widetilde{\varphi}_{\tau}$, respectively, and these extremum points should be inside the feasible region $\left[0, \tau_{\Delta}\right]$. It is easy to obtain that

$$
\begin{aligned}
& T_{\varphi}= \begin{cases}\emptyset, & \text { if } a_{\tau}>0, \\
\left\{\widehat{\tau}_{1}\right\}, & \text { if } a_{\tau}=0, \\
\left\{\tau_{2}\right\}, & \text { otherwise, }\end{cases} \\
& T_{\widetilde{\varphi}}= \begin{cases}\left\{\tau_{3}\right\}, & \text { if } \tilde{a}_{\tau}>0, \\
\left\{\widehat{\tau}_{3}\right\}, & \text { if } \widetilde{a}_{\tau}=0, \\
\left\{\tau_{3}, \tau_{4}\right\}, & \text { if }-\left(\widetilde{c}_{k}^{T} \widetilde{g}_{k}\right)^{2}<\widetilde{a}_{\tau}<0, \\
\emptyset, & \text { otherwise, }\end{cases}
\end{aligned}
$$


where

$$
\begin{aligned}
& \widehat{\tau}_{1}=\frac{\tilde{g}_{k}^{T} \widetilde{g}_{k}}{-b_{\tau}}, \\
& \tau_{1}=\frac{-b_{\tau}+\sqrt{\Delta_{\tau}}}{2 a_{\tau}}, \\
& \tau_{2}=\frac{-b_{\tau}-\sqrt{\Delta_{\tau}}}{2 a_{\tau}}, \\
& \widehat{\tau}_{3}=\frac{1}{2 \widetilde{c}_{k}^{T} \widetilde{g}_{k}}, \\
& \tau_{3}=\frac{-\widetilde{c}_{k}^{T} \widetilde{g}_{k}+\sqrt{\widetilde{\Delta}_{\tau}}}{\tilde{a}_{\tau}}, \\
& \tau_{4}=\frac{-\widetilde{c}_{k}^{T} \widetilde{g}_{k}-\sqrt{\widetilde{\Delta}_{\tau}}}{\tilde{a}_{\tau}}, \\
& \Delta_{\tau}=b_{\tau}^{2}-4 a_{\tau} \tilde{g}_{k}^{T} \widetilde{g}_{k}, \\
& \widetilde{\Delta}_{\tau}=\left(\widetilde{c}_{k}^{T} \widetilde{g}_{k}\right)^{2}+\widetilde{a}_{\tau} .
\end{aligned}
$$

Now, we have the the following conclusions.

Remark 2. Suppose that (5) and (35) hold. (i) If $\tilde{a}_{\tau} \geqslant 0$, then from (34) we know that $a_{\tau}>0$. (ii) If $-\left(\widetilde{c}_{k}^{T} \widetilde{g}_{k}\right)^{2} \leqslant \widetilde{a}_{\tau}<0, a_{\tau}=$ 0 , then $\widehat{\tau}_{1}>\tau_{\Delta}$. From (37) and (33), we have

$$
1-\widehat{\tau}_{1} \widetilde{c}_{k}^{T} \widetilde{g}_{k}=\frac{\tilde{a}_{\tau}+\left(\tilde{c}_{k}^{T} \widetilde{g}_{k}\right)^{2}}{\tilde{a}_{\tau}} \leqslant 0,
$$

where $\widehat{\tau}_{1}$ is defined in (37). However, from (37) and (35) we have $\widehat{\tau}_{1}>0$. We assume $\widehat{\tau}_{1} \in\left(0, \tau_{\Delta}\right]$; then, from (5) and (27) we have

$$
1-\widehat{\tau}_{1} \widetilde{c}_{k}^{T} \widetilde{g}_{k} \geqslant 1-\tau_{\Delta}\left\|\widetilde{c}_{k}\right\|\left\|\tilde{g}_{k}\right\| \geqslant 1-\epsilon_{1}>0,
$$

where the last inequality is obtained by (21) and this conflicts with (40). Therefore, $\widehat{\tau}_{1}>\tau_{\Delta}$.

Similarly, we can prove that if $-\left(\widetilde{c}_{k}^{T} \widetilde{g}_{k}\right)^{2} \leqslant \tilde{a}_{\tau}<0, a_{\tau}<0$, then $\tau_{2}>\tau_{\Delta}$.

Then, combining with (36) and Remark 2., we define a set

$$
T= \begin{cases}\left\{\tau_{3}\right\}, & \text { if } \tilde{a}_{\tau}>0, \\ \left\{\widehat{\tau}_{3}\right\}, & \text { if } \tilde{a}_{\tau}=0, \\ \left\{\tau_{3}, \tau_{4}\right\}, & \text { if }-\left(\widetilde{c}_{k}^{T} \widetilde{g}_{k}\right)^{2}<\widetilde{a}_{\tau}<0, \\ \left\{\widehat{\tau}_{1}\right\}, & \text { if } \widetilde{a}_{\tau}<-\left(\widetilde{c}_{k}^{T} \widetilde{g}_{k}\right)^{2}, \widetilde{a}_{\tau}=0, \\ \left\{\tau_{2}\right\}, & \text { if } \tilde{a}_{\tau}<-\left(\tilde{c}_{k}^{T} \widetilde{g}_{k}\right)^{2}, \tilde{a}_{\tau}<0, \\ \emptyset, & \text { otherwise, }\end{cases}
$$

where $\widehat{\tau}_{1}, \tau_{2}, \widehat{\tau}_{3}, \tau_{3}$, and $\tau_{4}$ are determined by (37)-(39).

Hence, it is easy to get the following theorems.
Theorem 3. Suppose that (5) and (35) hold. Then, the solution of (25) is

$$
\tau_{\mathrm{cp}}=\arg \min \left\{\widehat{\psi}(\tau), \tau \in T^{*}\right\}
$$

where

$$
T^{*}=\left(T \cap\left\{\left[0, \tau_{\Delta}\right]\right\}\right) \cup\left\{0, \tau_{\Delta}\right\}
$$

and $T$ is defined by (36).

Therefore, the steepest descent point $u_{\mathrm{cp}}=-\tau_{\mathrm{cp}} g$ is an approximate solution of the fractional trust region subproblem (18)-(19), where $\tau_{\mathrm{cp}}$ is defined by (43).

Similarly, the fractional trust region subproblem (18)(19) has the following property. The proof of this theorem is similar to Theorem 3.1 in [14], so we omit its proof.

Theorem 4. Suppose that (5) and (35) hold, where $\epsilon_{1} \epsilon$ $(0,1 / 3)$. If $\left\|u_{N}\right\|>\widetilde{\Delta}_{k}$, then the optimal solution of (18)-(19) must be on the boundary of trust region, where $u_{N}$ is defined in Subalgorithm 3.1.

In order to propose a generalized dogleg method of (18)(19), we set

$$
u(t)=t u_{N}+(1-t) u_{\mathrm{cp}}
$$

and calculate $t^{*}$ such that $\left\|u\left(t^{*}\right)\right\|=\widetilde{\Delta}_{k}$. Denote

$$
\begin{aligned}
& d=\left\|u_{N}-u_{\mathrm{cp}}\right\|^{2}, \\
& e=\left(u_{N}-u_{\mathrm{cp}}\right)^{T} u_{\mathrm{cp}}, \\
& f=\left\|u_{\mathrm{cp}}\right\|^{2}-\widetilde{\Delta}_{k}^{2} .
\end{aligned}
$$

If $\left\|u_{\mathrm{cp}}\right\|<\widetilde{\Delta}_{k}$, then there exist two real roots $t_{1}$ and $t_{2}$ :

$$
\begin{aligned}
& t_{1}=\frac{-e+\sqrt{e^{2}-d f}}{d}, \\
& t_{2}=\frac{-e-\sqrt{e^{2}-d f}}{d},
\end{aligned}
$$

where $0<t_{1}<1$ and $t_{2}<0$ (see $\left.[14,15]\right)$.

Based on the preceding theorems and analysis, we now give a generalized dogleg algorithm for solving (18)-(19).

Algorithm 5.

Step 1. Compute $u_{N}$ by Subalgorithm 3.1.

Step 2. If $\left\|u_{N}\right\| \leqslant \widetilde{\Delta}_{k}$, then $u_{k}=u_{N}$, and stop.

Step 3. If $\xi_{a}<0$, then compute $b_{\tau}$ as defined in (32), and go to Step 4. Otherwise, go to Step 5.

Step 4. If $b_{\tau} \geqslant 0$ or $\widetilde{c}_{k}^{T} \widetilde{g}_{k} \leqslant 0$, go to Step 5. Otherwise, compute $u_{\mathrm{cp}}=-\tau_{\mathrm{cp}} \widetilde{g}_{k}$, where $\tau_{\mathrm{cp}}$ is defined by (43). If $\tau_{\mathrm{cp}}=\tau_{\Delta}$, where 
$\tau_{\Delta}$ is defined by (27), then $u_{k}=u_{\text {cp }}$, and stop. If $\tau_{\text {cp }}=0$, go to Step 5. Otherwise, go to Step 6.

Step 5. Set $\widetilde{b}_{k}=\widetilde{c}_{k}=0$ and compute

$$
\begin{aligned}
& c_{a g}=\tilde{g}_{k}^{T} \widetilde{B}_{k} \widetilde{g}_{k}-\widetilde{a}_{k}^{T} \widetilde{g}_{k} \widetilde{g}_{k}^{T} \widetilde{g}_{k}, \\
& \widetilde{\tau}_{\mathrm{cp}}=\frac{\widetilde{g}_{k}^{T} \widetilde{g}_{k}}{c_{a g}}, \\
& u_{d}=-\widetilde{\tau}^{*} \widetilde{g}_{k},
\end{aligned}
$$

where

$$
\tilde{\tau}^{*}= \begin{cases}\tau_{\Delta}, & \text { if } c_{a g} \leqslant 0 \\ \min \left\{\tau_{\Delta}, \tilde{\tau}_{c p}\right\}, & \text { if } c_{a g}>0 .\end{cases}
$$

If $\widetilde{\tau}^{*}=\tau_{\Delta}$, then $u_{k}=u_{d}$, and stop. Otherwise, calculate $u_{\mathrm{cp}}=$ $-\widetilde{\tau}_{\mathrm{cp}} \widetilde{g}_{k}$, go to Step 6 .

Step 6. Calculate $t_{1}$ and $t_{2}$ as defined in (47); then,

$$
t^{*}=\arg \min \left\{\widetilde{\psi}_{k}(u(0)), \widetilde{\psi}_{k}\left(u\left(t_{1}\right)\right), \widetilde{\psi}_{k}\left(u\left(t_{2}\right)\right)\right\},
$$

where $u(t)$ is defined by (45). $u_{k}=u\left(t^{*}\right)$, and stop.

Then, we give the predicted decent bound in each iteration, which is the lower bound of the predicted reduction in each iteration:

$$
\operatorname{pred}_{k}(u)=\widetilde{\psi}_{k}(0)-\widetilde{\psi}_{k}(u)
$$

where $\widetilde{\psi}_{k}(u)$ is defined by (18).

Theorem 6. Suppose that (5) and (35) hold, where $\epsilon_{1} \in(0$, $1 / 3)$. If $u_{k}$ is obtained by Algorithm 5 , then

$$
\operatorname{pred}\left(u_{k}\right) \geqslant \frac{1}{2} \frac{\left\|\widetilde{g}_{k}\right\|}{\left\|\widetilde{B}_{k}\right\|} \min \left\{\left\|\widetilde{g}_{k}\right\|, \frac{\xi \widetilde{\Delta}_{k}}{\left\|\widetilde{B}_{k}^{-1}\right\|}\right\} \text {, }
$$

where $\xi=\left(1-\epsilon_{1}\right) /\left(1+\epsilon_{1}\right)^{2}$. ted.

This theorem is similar to that in [9] and its proof is omit-

\section{New Quasi-Newton Algorithm and Its Global Convergence}

In this section, we propose a quasi-Newton method with a fractional model for linearly equality constrained optimization and prove its convergence under some reasonable conditions. In order to solve problem (1)-(2), we consider the fractional model approximation for $f(x)$ about $u=0$; that is

$$
m_{k}(u)=f_{k}+\widetilde{\psi}_{k}(u)
$$

where $x=x_{k}+s=x_{k}+Q_{2} u, f_{k}=f\left(x_{k}\right)$, and $\widetilde{\psi}_{k}(u)$ is defined as (18). Thus, $m_{k}(0)=f_{k}, \widetilde{g}_{k}$, and $\widetilde{B}_{k}$ are the corresponding gradient and Hessian approximations of the function at the $k$ th iteration. We choose $u_{k}$ to minimize $m_{k}(u)$. There is a unique minimizer if and only if $\widetilde{B}_{k}$ is positive definite. In the following, we give our algorithm. If the current iteration is the feasible point $x_{k}$, then an equivalent form of (1)-(2) is to solve the reduced unconstrained problem

$$
\min _{u \in R^{n-m}} \tilde{f}(u)=f\left(x_{k}+Q_{2} u\right) .
$$

In the following, we consider the choice of the parameter vectors $\tilde{a}_{k}, \widetilde{b}_{k}$, and $\widetilde{c}_{k}$. We choose these vectors such that (53) satisfies the following conditions:

$$
\begin{aligned}
m_{k}(0) & =\widetilde{f}_{k}, \\
\nabla m_{k}(0) & =\widetilde{g}_{k}, \\
m_{k}\left(-u_{k-1}\right) & =\widetilde{f}_{k-1}, \\
\nabla m_{k}\left(-u_{k-1}\right) & =\widetilde{g}_{k-1},
\end{aligned}
$$

where

$$
\begin{aligned}
x_{k} & =x_{k-1}+s_{k-1}=x_{k-1}+Q_{2} u_{k-1}, \\
\tilde{f}_{k} & =\widetilde{f}(0)=f\left(x_{k}\right)=f_{k}, \\
\tilde{f}_{k-1} & =\widetilde{f}\left(-u_{k-1}\right)=f\left(x_{k-1}\right)=f_{k-1}, \\
\widetilde{g}_{k-1} & =Q_{2}^{T} g_{k-1} .
\end{aligned}
$$

Obviously, (55) holds. Then, from (56), we have

$$
\begin{aligned}
f_{k-1}= & f_{k}-\frac{\left(1-\widetilde{c}_{k}^{T} u_{k-1}\right) \tilde{g}_{k}^{T} u_{k-1}}{\widetilde{v}_{k-1}} \\
& +\frac{\left(1-\widetilde{c}_{k}^{T} u_{k-1}\right)^{2} u_{k-1}^{T} \widetilde{B}_{k} u_{k-1}}{2 \widetilde{v}_{k-1}^{2}}, \\
\tilde{g}_{k-1}= & \frac{1}{\widetilde{v}_{k-1}^{3}} \widetilde{Q}_{k-1}\left[\widetilde{v}_{k-1} \widetilde{g}_{k}-\left(1-\widetilde{c}_{k}^{T} u_{k-1}\right) \widetilde{B}_{k} u_{k-1}\right],
\end{aligned}
$$

where $\widetilde{v}_{k-1}=\left(1+\tilde{a}_{k}^{T} u_{k-1}\right)\left(1+\widetilde{b}_{k}^{T} u_{k-1}\right)$ and

$$
\begin{gathered}
\widetilde{Q}_{k-1}=\left(1-\widetilde{c}_{k}^{T} u_{k-1}\right) \widetilde{v}_{k-1} I-\left[\widetilde{c}_{k} \widetilde{v}_{k-1}+\left(1-\widetilde{c}_{k}^{T} u_{k-1}\right)\right. \\
\left.\cdot\left(\widetilde{a}_{k}\left(1+\widetilde{b}_{k}^{T} u_{k-1}\right)+\widetilde{b}_{k}\left(1+\tilde{a}_{k}^{T} u_{k-1}\right)\right)\right] u_{k-1}^{T} .
\end{gathered}
$$

If we choose

$$
\begin{aligned}
& \widetilde{a}_{k}=k_{1} \widetilde{g}_{k-1}, \\
& \widetilde{b}_{k}=k_{2} \widetilde{B}_{k-1} u_{k-1}, \\
& \widetilde{c}_{k}=k_{3} \widetilde{g}_{k},
\end{aligned}
$$


then these unknown parameters $k_{1}, k_{2}$, and $k_{3}$ can be obtained from (58)-(59). In the following, we give the derivation process of $k_{1}, k_{2}$, and $k_{3}$. First, we define some notations:

$$
\begin{aligned}
& \alpha_{k}=\tilde{g}_{k-1}^{T} u_{k-1}, \\
& \widetilde{\alpha}_{k}=\widetilde{g}_{k-1}^{T} \xi_{1}, \\
& \widehat{\alpha}_{k}=\tilde{g}_{k-1}^{T} \xi_{2}, \\
& \beta_{k}=u_{k-1}^{T} \widetilde{B}_{k-1} u_{k-1}, \\
& \widetilde{\beta}_{k}=u_{k-1}^{T} \widetilde{B}_{k-1} \xi_{1}, \\
& \widehat{\beta}_{k}=u_{k-1}^{T} \widetilde{B}_{k-1} \xi_{2}, \\
& \zeta_{k}=u_{k-1}^{T} \widetilde{B}_{k} u_{k-1}, \\
& \widetilde{\zeta}_{k}=u_{k-1}^{T} \widetilde{B}_{k} \xi_{1}, \\
& \widehat{\zeta}_{k}=u_{k-1}^{T} \widetilde{B}_{k} \xi_{2}, \\
& \gamma_{k}=\widetilde{g}_{k}^{T} u_{k-1}, \\
& \widetilde{\gamma}_{k}=\widetilde{g}_{k}^{T} \xi_{1}, \\
& \widehat{\gamma}_{k}=\widetilde{g}_{k}^{T} \xi_{2},
\end{aligned}
$$

where the vectors $\xi_{1}$ and $\xi_{2}$ are chosen to satisfy

$$
\tilde{\alpha}_{k}=\tilde{\gamma}_{k}=\widehat{\zeta}_{k}=\widehat{\gamma}_{k}=0 .
$$

For convenience, we omit the index $k$ of $\alpha_{k}, \widetilde{\alpha}_{k}, \widehat{\alpha}_{k}, \beta_{k}, \ldots, \widetilde{\gamma}_{k}$ and $\hat{\gamma}_{k}$.

On one hand, from (58) we have

$$
1-\widetilde{c}_{k}^{T} u_{k-1}=\eta \widetilde{v}_{k-1},
$$

where

$$
\eta=\frac{\gamma+\sqrt{\gamma^{2}+2 \zeta\left(\tilde{f}_{k-1}-\tilde{f}_{k}\right)}}{\zeta} .
$$

If the sequence $\left\{\tilde{f}_{k}\right\}$ is monotonically decreasing and $\widetilde{B}_{k}$ is positive definite, then we know that $\eta>0$ and (64) becomes

$$
1-k_{3} \gamma=\eta\left(1+k_{1} \alpha\right)\left(1+k_{2} \beta\right) \text {. }
$$

On the other hand, by left-multiplying $\xi_{1}^{T}$ on (59) and combining with (63), we have

$$
\begin{aligned}
& \left(1-k_{3} \gamma\right)\left(k_{2}(\tilde{\beta} \zeta-\beta \widetilde{\zeta})-\tilde{\zeta}\right) \\
& \quad-\left(1+k_{1} \alpha\right)\left(1+k_{2} \beta\right) k_{2} \tilde{\beta} \gamma=0 .
\end{aligned}
$$

Then, from (66), we have

$$
k_{2}=\frac{\eta \widetilde{\zeta}}{\ddot{\gamma}},
$$

where

$$
\ddot{\gamma}=\eta \widetilde{\beta} \zeta-\eta \beta \widetilde{\zeta}-\widetilde{\beta} \gamma \text {. }
$$

Similarly, by left multiplying $\xi_{2}^{T}$ on (59), from (63) and (66) we have

$$
k_{1}=\frac{-\left((\widehat{\alpha} \beta+\tau \tilde{\imath} \widehat{\beta}) k_{2}+\widehat{\alpha}\right)}{(\alpha \widehat{\alpha} \beta+\widetilde{i \eta}(\widehat{\alpha} \beta+\alpha \widehat{\beta})) k_{2}+\alpha \widehat{\alpha}+\widehat{\alpha} \widetilde{l \eta}},
$$

where $\tilde{\imath}=\gamma-\eta \zeta$. Substituting (68) into the above equation, we have

$$
k_{1}=-\frac{i}{\dot{\gamma}},
$$

where

$$
\begin{aligned}
& i=\widetilde{\imath}\left(\widehat{\alpha} \widetilde{\beta}-\eta^{2} \widetilde{\zeta} \widehat{\beta}\right), \\
& \dot{\gamma}=\widehat{\alpha \imath}+\eta \widehat{\alpha} \widetilde{\beta} \iota^{2} .
\end{aligned}
$$

And then from (66), we have

$$
k_{3}=\frac{1-\eta\left(1+k_{1} \alpha\right)\left(1+k_{2} \beta\right)}{\gamma} .
$$

Now we give the new quasi-Newton algorithm based on the fractional model (53).

\section{Algorithm 7.}

Step 0. Choose $x_{0} \in R^{n}, \epsilon_{1} \in(0,1 / 3), \varepsilon>0, \Delta_{\max }>0,0<$ $\iota_{1}<\iota_{2}<1,0<\delta_{1}<1<\delta_{2}, 0<\iota<1, \widetilde{B}_{0}=I$, and the initial trust region radius $\Delta_{0} \in\left(0, \Delta_{\max }\right]$. Compute $Q_{2}$ as defined in (15). Set $k=0$.

Step 1 (stopping criterion). Compute $f_{k}=f\left(x_{k}\right), g_{k}=$ $\nabla f\left(x_{k}\right)$, and $\tilde{g}_{k}=Q_{2}^{T} g_{k}$. If $\left\|\tilde{g}_{k}\right\| \leqslant \varepsilon$, then $x_{*}=x_{k}$, and stop. If $k=0$, go to Step 3 .

Step 2. Compute $y_{k-1}=\widetilde{g}_{k}-\widetilde{g}_{k-1}$. Update $\widetilde{B}_{k}$ by

$$
\widetilde{B}_{k}=\widetilde{B}_{k-1}-\frac{\widetilde{B}_{k-1} u_{k-1} u_{k-1}^{T} \widetilde{B}_{k-1}}{u_{k-1}^{T} \widetilde{B}_{k-1} u_{k-1}}+\frac{z_{k-1} z_{k-1}^{T}}{z_{k-1}^{T} u_{k-1}},
$$

where

$$
z_{k-1}=\theta y_{k-1}+(1-\theta) \widetilde{B}_{k-1} u_{k-1}, \quad \theta \in[0,1],
$$

$\theta$

$$
= \begin{cases}1, & \text { if } y_{k-1}^{T} u_{k-1} \geqslant 0.2 u_{k-1}^{T} \widetilde{B}_{k-1} u_{k-1}, \\ \frac{0.8 u_{k-1}^{T} \widetilde{B}_{k-1} u_{k-1}}{u_{k-1}^{T} \widetilde{B}_{k-1} u_{k-1}-y_{k-1}^{T} u_{k-1}}, & \text { otherwise. }\end{cases}
$$


Step 3. If $k \leqslant 1$, then set $\widetilde{a}_{k}=\widetilde{b}_{k}=\widetilde{c}_{k}=0, \widetilde{B}_{k}=Q_{2}^{T} B_{k} Q_{2}$, and $d_{k}=-\widetilde{B}_{k}^{-1} \widetilde{g}_{k}$, compute $\alpha_{k}$ such that Wolfe-Powell conditions are satisfied, and set $x_{k+1}=x_{k}+s_{k}=x_{k}+\alpha_{k} d_{k}$ and $u_{k}=Q_{2}^{T} s_{k}$, $k=k+1$, and go to Step 1 .

Step 4 . By the parameters $\widetilde{g}_{k-1}, \widetilde{g}_{k}$, and $\widetilde{B}_{k} u_{k-1}$ get $\xi_{1}$ and $\xi_{2}$.

Step 5. Compute $\alpha, \widetilde{\alpha}, \widehat{\alpha}, \ldots, \gamma, \widetilde{\gamma}$ and $\widehat{\gamma}$ as defined in (62). If $\gamma=0$, then set $\widetilde{b}_{k}=\widetilde{c}_{k}=0$. Calculate

$$
\begin{gathered}
\rho_{k}=\left(\tilde{f}_{k-1}-\tilde{f}_{k}\right)^{2}-\left(\tilde{g}_{k-1}^{T} u_{k-1}\right)\left(\tilde{g}_{k}^{T} u_{k-1}\right), \\
\dot{\beta}= \begin{cases}\frac{\left(\tilde{f}_{k-1}-\tilde{f}_{k}\right)+\sqrt{\rho_{k}}}{-\tilde{g}_{k-1}^{T} u_{k-1}}, & \text { if } \rho_{k} \geqslant 0, \\
1, & \text { otherwise }\end{cases}
\end{gathered}
$$

and set

$$
\widetilde{a}_{k}=\frac{1-\dot{\beta}}{\widetilde{g}_{k-1}^{T} u_{k-1}} \widetilde{g}_{k-1} .
$$

Otherwise, compute $\dot{\gamma}$ and $\ddot{\gamma}$ as defined in (72) and (69). If $\dot{\gamma}=0$ or $\ddot{\gamma}=0$, then set $\widetilde{a}_{k}=\widetilde{b}_{k}=\widetilde{c}_{k}=0$. Otherwise, calculate $\widetilde{a}_{k}, \widetilde{b}_{k}$, and $\widetilde{c}_{k}$, where $k_{1}, k_{2}$, and $k_{3}$ in (61) are determined by (71), (68), and (73).

Step 6. If $\left\|\widetilde{a}_{k}\right\|>\epsilon_{1} / \widetilde{\Delta}_{k}$, then $\widetilde{a}_{k}=\epsilon_{1} a_{k} / \widetilde{\Delta}_{k}\left\|a_{k}\right\|$. Update $\widetilde{b}_{k}$ and $\widetilde{c}_{k}$ with the same way such that (5) are satisfied.

Step 7. By the parameters $\widetilde{a}_{k}, \widetilde{b}_{k}, \widetilde{c}_{k}, \widetilde{g}_{k}$, and $\widetilde{\Delta}_{k}$, solve the subproblem (18)-(19) by Algorithm 5 to get $u_{k}$. Set $s_{k}=Q_{2} u_{k}$.

Step 8. Compute

$$
\rho_{k}=\frac{\operatorname{Ared}\left(u_{k}\right)}{\operatorname{Pred}\left(u_{k}\right)}
$$

where

$$
\begin{aligned}
& \operatorname{Ared}\left(u_{k}\right)=\operatorname{Ared}\left(s_{k}\right)=f\left(x_{k}\right)-f\left(x_{k}+s_{k}\right), \\
& \operatorname{Pred}\left(u_{k}\right)=m_{k}(0)-m_{k}\left(u_{k}\right)=-\widetilde{\psi}_{k}\left(u_{k}\right) .
\end{aligned}
$$

Step 9. Update the trust region radius:

$$
\widetilde{\Delta}_{k+1}= \begin{cases}\delta_{1} \tilde{\Delta}_{k}, & \text { if } \rho_{k} \leqslant \iota_{1}, \\ \min \left\{\delta_{2} \widetilde{\Delta}_{k}, \Delta_{\max }\right\}, & \text { if } \rho_{k} \geqslant \iota_{2},\left\|u_{k}\right\|=\widetilde{\Delta}_{k}, \\ \tilde{\Delta}_{k}, & \text { otherwise. }\end{cases}
$$

Step 10. If $\rho_{k} \geqslant \imath_{1}$, then $x_{k+1}=x_{k}+s_{k}$. Set $k=k+1$, and go to Step 1. Otherwise, $x_{k+1}=x_{k}, k=k+1$, and go to Step 6 .
Next we present the global convergence theorem which says the reduced gradients converge to zero.

Theorem 8. Assume that (5) and (35) hold, where $\epsilon_{1} \in(0$, $1 / 3)$. If $f$ is continuously differentiable and bounded below in some set containing all iterations generated by Algorithm 7 , the sequences $\left\{\left\|\widetilde{g}_{k}\right\|\right\}$ and $\left\{\left\|\widetilde{B}_{k}\right\|\right\}$ are uniformly bounded. Then,

$$
\liminf _{k \rightarrow \infty}\left\|\widetilde{g}_{k}\right\|=0
$$

Proof. Assume that the theorem is false and there is $\bar{\varepsilon}>0$ such that $\widetilde{g}_{k} \geqslant \bar{\varepsilon}$ for all $k$. From the assumption, we can assume that

$$
\begin{aligned}
\left\|\widetilde{a}_{k}\right\| & \leqslant \bar{a}, \\
\left\|\widetilde{b}_{k}\right\| & \leqslant \bar{b}, \\
\left\|\widetilde{c}_{k}\right\| & \leqslant \bar{c}, \\
\left\|\widetilde{g}_{k}\right\| & \leqslant \bar{g}, \\
0 & <\left\|\widetilde{B}_{k}\right\| \leqslant \bar{B}, \\
0 & <\left\|\widetilde{B}_{k}^{-1}\right\| \leqslant \widetilde{B}
\end{aligned}
$$

hold for all $k$. From (52) and the assumptions in the theorem, we have

$$
\operatorname{pred}\left(s_{k}\right) \geqslant \frac{\bar{\varepsilon}}{2 \bar{B}} \min \left\{\bar{\varepsilon}, \frac{\xi \widetilde{\Delta}_{k}}{\widetilde{B}}\right\} \geqslant c_{1} \min \left\{\widetilde{\Delta}_{k}, c_{2}\right\}
$$

where $c_{1}$ and $c_{2}$ are some positive constants. Then, from Step 10 of Algorithm 7, we have

$$
f_{k}-f_{k+1} \geqslant \iota_{1} \operatorname{pred}\left(s_{k}\right) \geqslant \iota_{1} c_{1} \min \left\{\widetilde{\Delta}_{k}, c_{2}\right\} .
$$

Since $f(x)$ is bounded from below and $f_{k+1}<f_{k}$ for all $k$, we have that $\sum_{k=1}^{\infty} \min \left\{\widetilde{\Delta}_{k}, c_{2}\right\}$ is convergent, and $\widetilde{\Delta}_{k} \rightarrow 0$ as $k \rightarrow \infty$.

On the other hand, when $\widetilde{\Delta}_{k} \rightarrow 0,\left\|u_{k}\right\| \rightarrow 0$. From Step 6 of Algorithm 7, we have

$$
\begin{aligned}
& \left|\tilde{a}_{k}^{T} u_{k}\right| \leqslant\left\|\tilde{a}_{k}\right\| \widetilde{\Delta}_{k} \leqslant \epsilon_{1}, \\
& \left|\tilde{b}_{k}^{T} u_{k}\right| \leqslant \epsilon_{1},
\end{aligned}
$$

where $\epsilon_{1} \in(0,1 / 3)$. Thus, we have

$$
\begin{aligned}
& \frac{1}{1-\tilde{a}_{k}^{T} u_{k}}=1+\tilde{a}_{k}^{T} u_{k}+o\left(\left\|u_{k}\right\|\right), \\
& \frac{1}{1-\widetilde{b}_{k}^{T} u_{k}}=1+\widetilde{b}_{k}^{T} u_{k}+o\left(\left\|u_{k}\right\|\right) .
\end{aligned}
$$


TABLE 1: Test functions.

\begin{tabular}{lc}
\hline Pro. & Function name \\
\hline 1 & HS 9 \\
2 & HS 48 \\
3 & HS 49 \\
4 & HS 50 \\
5 & Chen 3.3.1 \\
6 & Chen 3.3.2 \\
7 & Cube \\
8 & Penalty-I \\
9 & Beale \\
10 & Conic \\
11 & Extended Powell \\
12 & Broyden Tridiagonal \\
13 & Rosenbrock \\
14 & Chained Freudenstein and Roth \\
15 & Extended Trigonometric \\
16 & Penalty-III \\
17 & Troesch \\
18 & Cragg and Levy \\
\hline
\end{tabular}

By computing, we obtain

$$
\begin{aligned}
& \frac{\left(1+\tilde{c}_{k}^{T} u_{k}\right) \widetilde{g}_{k}^{T} u_{k}}{\left(1-\widetilde{a}_{k}^{T} u_{k}\right)\left(1-\widetilde{b}_{k}^{T} u_{k}\right)}=g_{k}^{T} u_{k}+O\left(\left\|u_{k}\right\|^{2}\right) \\
& \frac{\left(1+\widetilde{c}_{k}^{T} u_{k}\right)^{2} u_{k}^{T} \widetilde{B}_{k} u_{k}}{\left(1-\widetilde{a}_{k}^{T} u_{k}\right)^{2}\left(1-\widetilde{b}_{k}^{T} u_{k}\right)^{2}}=u_{k}^{T} \widetilde{B}_{k} u_{k}+o\left(\left\|u_{k}\right\|^{2}\right) \\
& \operatorname{Ared}\left(u_{k}\right)=-g_{k}^{T} u_{k}-u_{k}^{T} \widetilde{B}_{k} u_{k}+o\left(\left\|u_{k}\right\|^{2}\right)
\end{aligned}
$$

Then, from (80), we have

$$
\operatorname{Pred}\left(u_{k}\right)=\operatorname{Ared}\left(u_{k}\right)+O\left(\left\|\widetilde{\Delta}_{k}\right\|^{2}\right),
$$

which indicates that

$$
\lim _{k \rightarrow \infty} \rho_{k}=1
$$

By the updating in Step 9 of Algorithm 7, we have $\widetilde{\Delta}_{k+1} \geqslant \widetilde{\Delta}_{k}$, which is a contradiction to $\widetilde{\Delta}_{k} \rightarrow 0$. The theorem is proved.

\section{Numerical Tests}

In this section, Algorithm 7 (abbreviated as FTR) is tested with some test problems which are chosen from [16, 17]. These test problems are listed in Table 1. We choose linear constrained problems HS9, HS48, HS49, HS50, Chen 3.3.1, and Chen 3.3.2. Moreover, in order to test Algorithm 7 more generally, we designed some problems where the objective functions are Pro. 7-18 (see $[14,18])$ and the linear equality constraints are Pro. 1-6. If $\widetilde{b}_{k}=\widetilde{c}_{k}=0$ in Algorithm 7, we can obtain the conic model algorithm and call this algorithm CTR. We solve the following 18 test problems by FTR and CTR and compare their results.

All the computations are carried out in Matlab R2012b on a microcomputer in double precision arithmetic. These tests use the same stopping criterion $\left\|\widetilde{g}_{k}\right\| \leqslant 10^{-4}$. The columns in the tables have the following meanings: Pro. denotes the numbers of the test problems; $n$ is the dimension of the test problems; Iter is the number of iterations; nf and ng are the numbers of function and gradient evaluations, respectively; $\|\widetilde{g}\|$ is the Euclidean norm of the final reduced gradient; $\mathrm{CPU}(\mathrm{s})$ denotes the total iteration time of the algorithm in seconds. The parameters in these algorithms are

$$
\begin{aligned}
B_{0} & =I, \\
\epsilon_{1} & =0.33, \\
\Delta_{0} & =1, \\
\Delta_{\max } & =10, \\
l & =0.15, \\
\mu & =0.85, \\
\iota_{1} & =0.25, \\
\iota_{2} & =0.75, \\
\delta_{2} & =4 .
\end{aligned}
$$

The numerical comparison for 18 small-scale test problems is listed in Table 2. We can see that FTR is better than CTR for 15 tests in the number of iterations and the remaining 3 tests are similar. Because FTR needs some extra algebra computation for some parameters, FTR takes more time than CTR for small problems.

The numerical results of some large-scale problems are presented in Table 3. From Table 3, we find that for large-scale problems the CPU time of FTR is approximately the same as that of CTR but it has fewer number of iterations. From the above comparison, we see that FTR is slightly more effective and robust for these large-scale test problems.

The fractional model in Algorithm 7 is the extension of conic model. By using more information of function and gradient from the previous iterations and choosing parameters flexibly, the fractional model can be more approximate to the original problem. And the global convergence of the proposed quasi-Newton trust region algorithm is also proved. Numerical experiment shows the algorithm is effective and robust, including for large-scale test problems. The theoretical results and the numerical results lead us to believe that the method is worthy of further study. For example, we can consider using fractional model to solve the nonlinear equality constrained optimization problem. 
TABLE 2: The numerical results of Algorithm 7 for some test problems.

\begin{tabular}{|c|c|c|c|c|c|c|c|}
\hline Pro. & $n$ & Starting point & Algorithm & Iter & $\mathrm{nf} / \mathrm{ng}$ & $\|g\|$ & $\mathrm{CPU}(\mathrm{s})$ \\
\hline \multirow{2}{*}{1} & \multirow{2}{*}{6} & \multirow{2}{*}{$(0,0,0,0,0,0)$} & CTR & 6 & $7 / 8$ & $7.559746(-5)$ & 0.084639 \\
\hline & & & FTR & 5 & $6 / 6$ & $1.693174(-5)$ & 0.161438 \\
\hline \multirow{2}{*}{2} & \multirow{2}{*}{5} & \multirow{2}{*}{$(3,5,-3,2,-2)$} & CTR & 13 & $14 / 9$ & $1.637781(-5)$ & 0.094287 \\
\hline & & & FTR & 8 & $9 / 9$ & $2.148387(-6)$ & 0.098268 \\
\hline \multirow{2}{*}{3} & \multirow{2}{*}{5} & \multirow{2}{*}{$(2,2,-9,3,3)$} & CTR & 27 & $28 / 21$ & $5.815109(-5)$ & 0.110429 \\
\hline & & & FTR & 25 & $26 / 17$ & $4.996278(-5)$ & 0.149000 \\
\hline \multirow{2}{*}{4} & \multirow{2}{*}{10} & $(0,-6,6,0,0$ & CTR & 12 & $13 / 13$ & $3.889891(-5)$ & 0.100359 \\
\hline & & $0,-6,6,0,0)$ & FTR & 8 & $9 / 9$ & $2.397640(-5)$ & 0.152117 \\
\hline \multirow{2}{*}{5} & \multirow{2}{*}{8} & $(0,3,-1,-2$ & CTR & 16 & $17 / 14$ & $7.255223(-5)$ & 0.099120 \\
\hline & & $0,3,-1,-2)$ & FTR & 16 & $17 / 17$ & $1.249467(-5)$ & 0.129272 \\
\hline \multirow{2}{*}{6} & \multirow{2}{*}{8} & $(7 / 3,11 / 3,-1,-1$ & CTR & 22 & $23 / 19$ & $3.128858(-6)$ & 0.101571 \\
\hline & & $7 / 3,11 / 3,-1,-1)$ & FTR & 21 & $22 / 19$ & $2.865070(-7)$ & 0.167233 \\
\hline \multirow{2}{*}{7} & \multirow{2}{*}{6} & \multirow{2}{*}{$(3,4,3,4,3,4)$} & CTR & 8 & $9 / 9$ & $2.208537(-7)$ & 0.093060 \\
\hline & & & FTR & 8 & $9 / 9$ & $1.765690(-8)$ & 0.139521 \\
\hline \multirow{2}{*}{8} & \multirow{2}{*}{6} & \multirow{2}{*}{$(3,4,3,4,3,4)$} & CTR & 14 & $15 / 11$ & $8.493218(-7)$ & 0.095873 \\
\hline & & & FTR & 12 & $13 / 10$ & $1.155176(-5)$ & 0.166608 \\
\hline \multirow{2}{*}{9} & \multirow{2}{*}{6} & \multirow{2}{*}{$(6,8,6,8,6,8)$} & CTR & 21 & $22 / 18$ & $1.208648(-6)$ & 0.094048 \\
\hline & & & FTR & 19 & $20 / 18$ & $3.705893(-5)$ & 0.170549 \\
\hline \multirow{2}{*}{10} & \multirow{2}{*}{6} & \multirow{2}{*}{$-(1.5,2,1.5,2,1.5,2)$} & CTR & 13 & $14 / 10$ & $1.789828(-6)$ & 0.104145 \\
\hline & & & FTR & 9 & $10 / 7$ & $4.017095(-6)$ & 0.158877 \\
\hline 11 & 8 & $(0304$ & CTR & 15 & $16 / 13$ & $3.013621(-5)$ & 0.087370 \\
\hline 11 & 0 & $(0.0,0.4$, & FTR & 7 & $8 / 8$ & $7.252083(-5)$ & 0.121116 \\
\hline 12 & 6 & $0304)$ & CTR & 26 & $27 / 16$ & $5.317180(-5)$ & 0.1160001 \\
\hline 12 & 0 & $(0.0,0.4, \ldots, 0.0,0.4)$ & FTR & 24 & $25 / 14$ & $5.917188(-5)$ & 0.187447 \\
\hline 13 & 6 & $(152152152)$ & CTR & 6 & $7 / 7$ & $8.707409(-5)$ & 0.081667 \\
\hline 10 & 0 & $(1.5,2,1.5,2,1.5,2)$ & FTR & 6 & $7 / 7$ & $5.998420(-5)$ & 0.115520 \\
\hline 14 & 6 & $(686868)$ & CTR & 34 & $35 / 20$ & $3.185767(-5)$ & 0.106515 \\
\hline 14 & 0 & $(0,0,0,0,0,0)$ & FTR & 22 & $23 / 23$ & $4.845919(-6)$ & 0.158603 \\
\hline 15 & 8 & $(0304 \quad 0304)$ & CTR & 10 & $11 / 9$ & $1.738762(-5)$ & 0.092062 \\
\hline & & $(0.5,0.4, \ldots, 0.5,0.4)$ & FTR & 9 & $10 / 8$ & $5.005195(-5)$ & 0.146342 \\
\hline 16 & 6 & $(03,04 \quad 03,04)$ & CTR & 13 & $14 / 12$ & $3.409181(-5)$ & 0.083445 \\
\hline & & $(0.0,0.4, \ldots, 0.0,0.4)$ & FTR & 12 & $13 / 12$ & $9.332787(-5)$ & 0.164178 \\
\hline 17 & 6 & $(0304 \quad 0304)$ & CTR & 29 & $30 / 17$ & $1.845528(-6)$ & 0.088997 \\
\hline 17 & 0 & $(0.0,0.4, \ldots, 0.5,0.4)$ & FTR & 19 & $20 / 17$ & $5.983264(-6)$ & 0.159851 \\
\hline 18 & 8 & $(0304 \quad 0304)$ & CTR & 12 & $13 / 9$ & $7.722466(-7)$ & 0.087020 \\
\hline & & $(0.3,0.4, \ldots, 0.3,0.4)$ & FTR & 9 & $10 / 9$ & $4.353866(-7)$ & 0.149733 \\
\hline
\end{tabular}

TABLE 3: The numerical results of Algorithm 7 for some test problems $(n=3000)$.

\begin{tabular}{lccccrr}
\hline Pro. & Starting point & Algorithm & Iter & nf/ng & $\|g\|$ & CPU (s) \\
\hline \multirow{2}{*}{7} & CTR & 25 & $26 / 26$ & $3.737202(-9)$ & 28.5976 \\
& $(6,8, \ldots, 6,8)$ & FTR & 21 & $22 / 22$ & $3.849243(-9)$ & 26.2795 \\
\hline \multirow{2}{*}{8} & $(6,8, \ldots, 6,8)$ & CTR & 12 & $13 / 12$ & $8.930053(-6)$ & 13.8295 \\
& & FTR & 12 & $13 / 11$ & $3.113077(-7)$ & $3.388081(-6)$ \\
\hline \multirow{2}{*}{9} & $(6,8, \ldots, 6,8)$ & CTR & 25 & $26 / 26$ & 28.7506 \\
\hline \multirow{2}{*}{10} & FTR & 24 & $1.176732(-6)$ & 30.7385 \\
\hline \multirow{2}{*}{11} & $(6,8, \ldots, 6,8)$ & CTR & 12 & $13 / 13$ & $1.226943(-7)$ & 14.9229 \\
& & FTR & 10 & $11 / 11$ & $6.842114(-6)$ & 14.9369 \\
\hline
\end{tabular}




\section{Competing Interests}

The authors have no competing interests regarding this paper.

\section{Acknowledgments}

This work was supported by the National Natural Science Foundation of China (Grant no. 11071117 and 71301060), the Natural Science Foundation of Jiangsu Province (BK20141409), Funding of Jiangsu Innovation Program for Graduate Education (KYZZ_0089) ("the Fundamental Research Funds for the Central Universities"), and the Humanistic and Social Science Foundation of Ministry of Education of China (12YJA630122).

\section{References}

[1] W. C. Davidon, "Conic approximations and collinear scalings for optimizers," SIAM Journal on Numerical Analysis, vol. 17, no. 2, pp. 268-281, 1980.

[2] R. Schnabel, "Conic methods for unconstrained minimization and tensor methods for nonlinear equations," in Mathematical Programming: The State of the Art, A. Bachem, M. Grötschel, and B. Korte, Eds., pp. 417-438, Springer, Heidelberg, Germany, 1982.

[3] D. C. Sorensen, "Newton's method with a model trust region modification," SIAM Journal on Numerical Analysis, vol. 19, no. 2, pp. 409-426, 1982.

[4] W. Sun and Y. X. Yuan, "A conic trust-region method for nonlinearly constrained optimization," Annals of Operations Research, vol. 103, pp. 175-191, 2001.

[5] C. X. Xu and X. Y. Yang, "Convergence of conic quasiNewton trust region methods for unconstrained minimization," Mathematical Application, vol. 11, no. 2, pp. 71-76, 1998.

[6] Y. X. Yuan, "A review of trust region algorithms for optimization," in Proceedings of the International Congress on Industrial and Applied Mathematics (ICIAM '00), vol. 99, pp. 271-282, 2000.

[7] D. M. Gay, "Computing optimal locally constrained steps," SIAM Journal on Scientific and Statistical Computing, vol. 2, no. 2, pp. 186-197, 1981.

[8] J.-M. Peng and Y.-X. Yuan, "Optimality conditions for the minimization of a quadratic with two quadratic constraints," SIAM Journal on Optimization, vol. 7, no. 3, pp. 579-594, 1997.

[9] H. L. Zhu, Q. Ni, and M. L. Zeng, "A quasi-Newton trust region method based on a new fractional model," Numerical Algebra, Control and Optimization, vol. 5, no. 3, pp. 237-249, 2015.

[10] W. Y. Sun, J. Y. Yuan, and Y. X. Yuan, "Conic trust region method for linearly constrained optimization," Journal of Computational Mathematics, vol. 21, no. 3, pp. 295-304, 2003.

[11] X. P. Lu and Q. Ni, "A trust region method with new conic model for linearly constrained optimization," Or Transactions, vol. 12, pp. 32-42, 2008.

[12] Q. Ni, Optimization Method and Program Design, Science Press, Beijing, China, 2009.

[13] L. W. Zhang and Q. Ni, "Trust region algorithm of new conic model for nonlinearly equality constrained optimization," Journal on Numerical Methods and Computer Applications, vol. 31, no. 4, pp. 279-289, 2010.
[14] M. F. Zhu, Y. Xue, and F. S. Zhang, "A quasi-Newton type trust region method based on the conic model," Numerical Mathematics, vol. 17, no. 1, pp. 36-47, 1995 (Chinese).

[15] X. P. Lu and Q. Ni, "A quasi-Newton trust region method with a new conic model for the unconstrained optimization," Applied Mathematics and Computation, vol. 204, no. 1, pp. 373-384, 2008.

[16] W. Hock and K. Schittkowski, Test Examples for Nonlinear Programming Codes, Springer, Berlin, Germany, 1981.

[17] X. Y. Chen, Research on the geometric algorithms for programs with constraints of linear equalities [M.S. thesis], Fujian Normal University, 2012.

[18] J. J. More, B. S. Garbow, and K. E. Hillstrom, “Testing unconstrained optimization software," ACM Transactions on Mathematical Software, vol. 7, no. 1, pp. 17-41, 1981. 


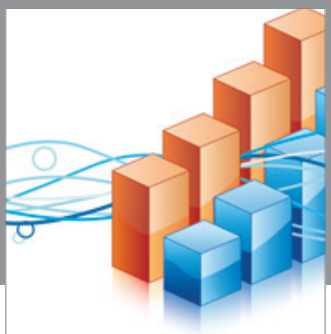

Advances in

Operations Research

vatem alat4

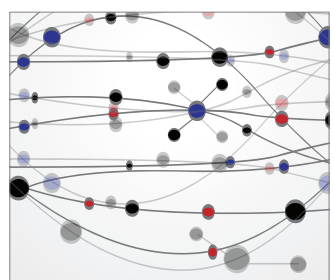

\section{The Scientific} World Journal
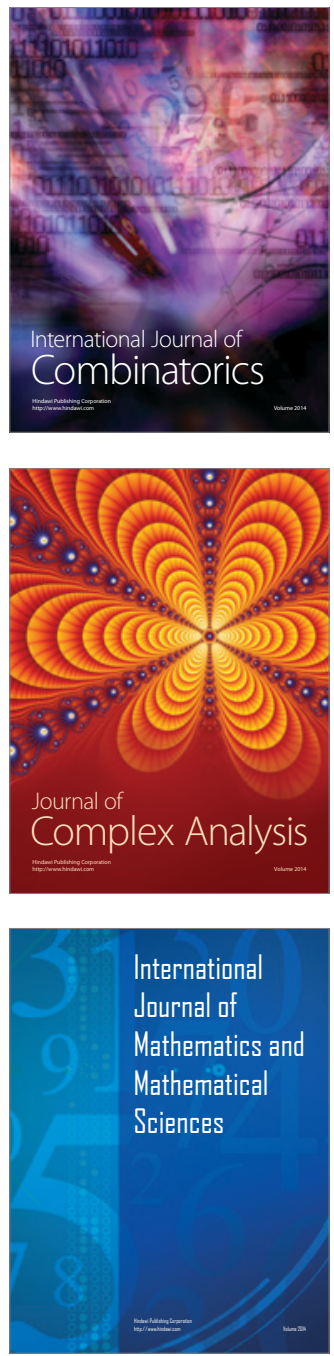
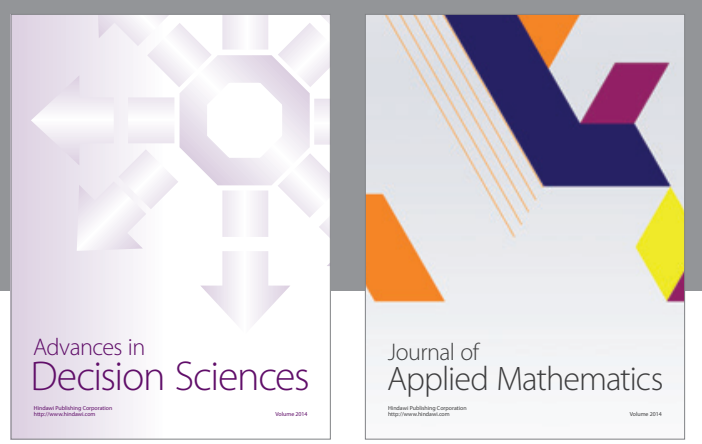

Algebra

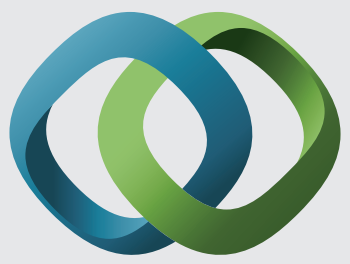

\section{Hindawi}

Submit your manuscripts at

http://www.hindawi.com
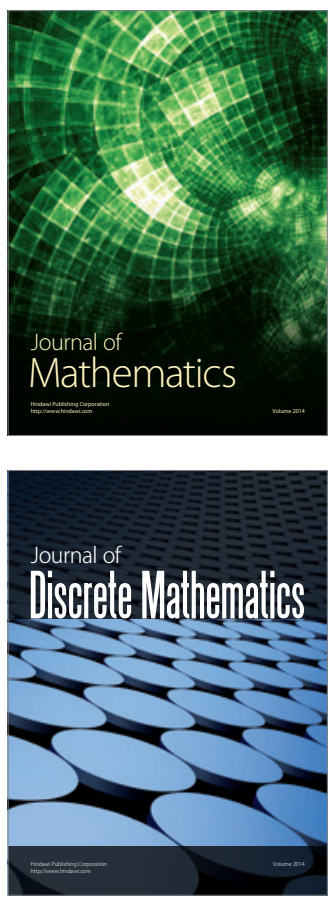

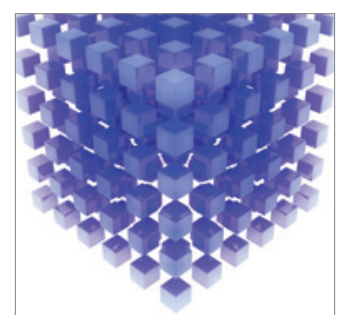

Mathematical Problems in Engineering
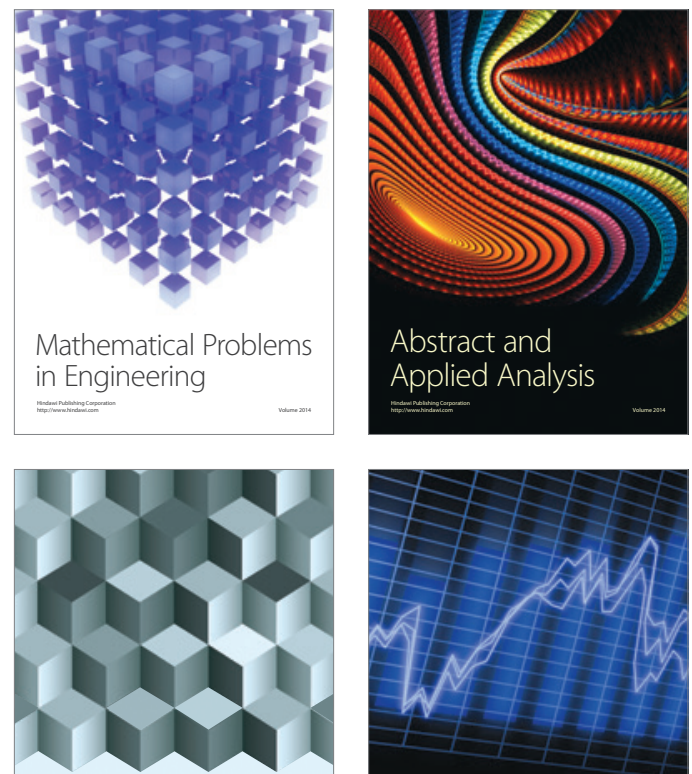

Journal of

Function Spaces

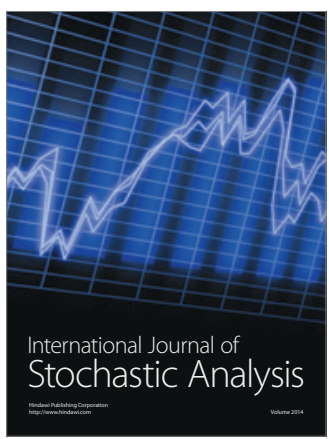

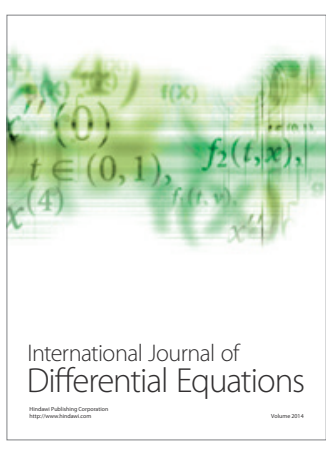
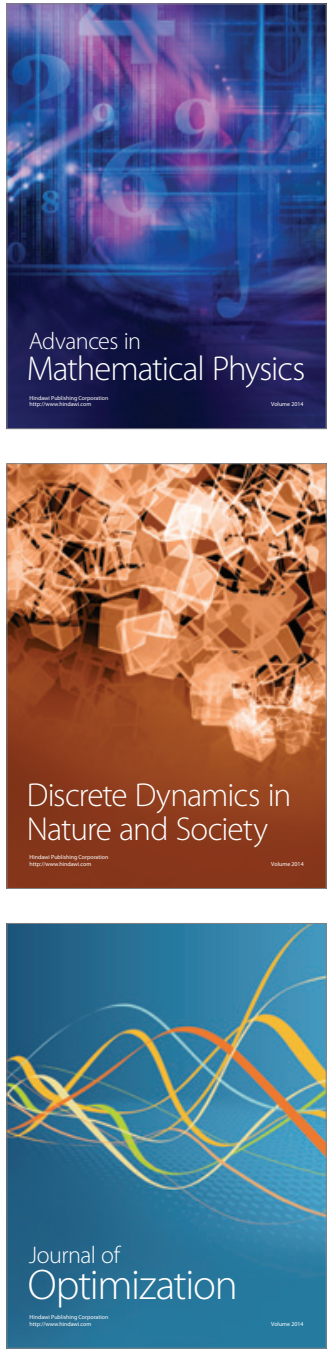